\title{
Changes in Renal Function Among Women With Preeclampsia in a Tertiary Health Institution in Nigeria
}

\author{
Njoku Charles ${ }^{1,2^{*}}$, Njoku Amarachukwu ${ }^{2}$, Edet Ekpo ${ }^{1,2}$, Emechebe Cajethan $^{1,2}$
}

\begin{abstract}
Objectives: Preeclampsia is a pregnancy-related multisystem medical disorder which adversely affects the health of the mother and fetus. Studies have shown varied changes in biochemical renal function indices in preeclampsia. These changes vary in different environments and affect renal health and pregnancy outcomes. Accordingly, this study aimed to determine changes in serum electrolytes, urea, and creatinine in women with preeclampsia in Calabar.

Materials and Methods: A cross-sectional comparative study was conducted on 144 pregnant women including 72 normotensive and 72 preeclamptic cases of similar age groups, with singleton pregnancies, and in the third trimester. The serum sodium, potassium, bicarbonate, chloride, urea, and creatinine were assayed in the two groups. Finally, statistical analysis was done using SPSS, version 22.

Results: Based on the results, the preeclamptic group had significantly higher mean blood pressure (BP) and body mass index (BMI) compared to the normotensives. In addition, women with preeclampsia had a significant decrease in serum potassium and an increase in the serum creatinine compared to the normotensive group. The results further revealed that serum potassium, as well as systolic BP and diastolic BP had a significant inverse correlation in preeclampsia $(P<0.01$ and $P<0.05$, respectively). Eventually, the serum creatinine had a significant positive relationship with systolic and diastolic BP $(P<0.01)$

Conclusions: In general, serum potassium and creatinine levels significantly altered in preeclampsia and were associated with increased disease severity. Therefore, it is suggested the serial electrolyte and creatinine profile should be used in the monitoring disease severity, which informs a timely intervention and reduces complications associated with preeclampsia.

Keywords: Electrolytes, Urea, Creatinine, Preeclampsia, Pregnancy outcome
\end{abstract}

\section{Introduction}

Preeclampsia is a multisystem disorder that is specific to pregnancy which affects $3-5 \%$ of pregnancies and negatively affects the health of the mother and fetus (13). Although its pathogenesis is unknown, the kidney plays a key role in its clinical manifestations (4). The most characteristic renal change in preeclampsia is marked as the cellular swelling of the glomerular endothelium, which is also called endotheliosis (5). The renal changes are characterized by glomerular endothelial cell injury, damage to the glomerular basement membrane, and the hypoperfusion of the glomeruli, resulting in proteinuria and the diminished glomerular filtration rate (5). Renal adaptations to these changes may manifest as elevated blood pressure (BP) (5).

In addition, preeclampsia is regarded as the "disease of theories" as there are many hypotheses that explain its pathophysiology. However, some new markers can predict the disease, including soluble fms-like tyrosine kinase 1, soluble endoglin, and the placenta growth factor (6). Estimating serum electrolytes, urea, and creatinine may provide a useful index for understanding physiological and pathological changes in preeclampsia. Studies have reported a significant increase in the serum urea and creatinine in preeclampsia (7). The obtained findings regarding electrolyte changes in preeclampsia are complex and contradictory. For instance, some studies reported no significant disparity while others observed significant changes in this regard (7-9). There seems to be no consensus on the renal parameter to be used as a marker for disease severity. Moreover, in our center, there is no data to represent the pattern of renal biochemical changes among pregnant women with preeclampsia regarding suggesting the possible markers of disease severity in our locality.

Considering the limitation of the screening options, the prevention of the disease would be beneficial. Among those at the risk of preeclampsia, the administration of lowdose aspirin in early pregnancy prevents the occurrence of the disease (10). Calcium supplements, antioxidants (i.e., vitamins $\mathrm{C}$ and $\mathrm{E}$ ), and vitamin $\mathrm{D}$ administration are other preventive measures against preeclampsia (10).

This study was carried out to determine how preeclampsia affects serum electrolytes, urea, and 
Key messages

- Study highlights significance of renal biochemical changes in severity of preeclampsia and may inform intervention to reduce mortality.

- Serum Potassium reduces in preeclampsia whereas serum creatinine increases in preeclampsia and these respective changes co-notes with progressive disease severity.

creatinine in preeclampsia and how these changes relate to the marker of disease severity in this environment. This may provide a platform for monitoring disease severity and timely intervention in order to prevent maternal and fetal complications.

\section{Materials and Methods}

This cross-sectional case-comparative study was performed on 144 pregnant women including 72 pregnant women with preeclampsia and 72 normotensive pregnant women as controls. Further, the study lasted for 18 months from May 1, 2017 to September 30, 2018. Before study initiation, the ethical approval for the study protocol was obtained from the Health Research and Ethics Committee of the University of Calabar Teaching Hospital, Calabar. Furthermore, informed consent was obtained from each participant and then a structured questionnaire was used to obtain data.

The study sample size was determined by the following formula.

Using the mean and standard deviation of the serum creatinine in preeclamptic and normotensive pregnant women, according to Manjareeka and Nanda, the power of $80 \%$, the significance level of $5 \%$, and the attrition rate of $10 \%$ (7) were considered based on the aim of the study. Some participants were on admission in the ward while some others were attendees in the antenatal clinic in the hospital. It should be noted that they were all within the same age range, with singleton pregnancies at 28 weeks of gestation or more. Women with a history of pre-existing renal disease, diabetes mellitus, urinary tract infection, and liver disease were excluded from the study. The height and weight of each participant were measured and their body mass indexes (BMIs) were calculated as well. Moreover, each participant was asked to rest for 5 minutes, then, sitting at an angle of 45 degrees, two BP measurements were taken at least 5 minutes apart and the sustained elevation was confirmed by repeating the measurement at least after 4 hours. Next, a dip-stick urinalysis was done for each participant in order to screen for proteinuria. Participants with preeclampsia (cases) were those with a BP of greater than or equal to $140 / 90 \mathrm{~mm} \mathrm{Hg}$ and proteinuria $2+$ while the study controls included those with a BP of less than 140/90 $\mathrm{mm} \mathrm{Hg}$ and no proteinuria.

Eight milliliters of the venous blood were collected from the antecubital vein of each participant without stasis and dispensed into a plastic vacuum plain bottle. Additionally, the blood in the plain bottle was allowed to clot and then centrifuged at a speed of $3000 \mathrm{rpm}$ for 10 minutes. The serum was aspirated and dispensed into plain tubes and stored at $-20^{\circ} \mathrm{C}$ as well. In addition, serum electrolytes were measured using the flame photometry method. Eventually, the serum urea and serum creatinine were measured using the Berthelot and Jaffes Kinetic methods, respectively.

Data analysis was done by SPSS statistics (IBM Corp., version 22) program. The differences between group means were determined using the analysis of variance. Finally, Pearson's correlation was used to analyze the correlation between the variables and the level of significance was below $5 \%$.

\section{Results}

Data on age, BP, BMI, serum electrolytes, serum creatinine, and serum urea are shown in Table 1 . The mean ages of the two groups were $30.5 \pm 4.18$ years and $30.69 \pm 4.54$ years for normotensive and preeclamptic women, respectively. Further, the mean BMI was $28.14 \pm 2.85$ and $30.96 \pm 2.04$ for normotensive pregnant women and pregnant women with preeclampsia, respectively $(P=0.03)$. Similarly, the mean serum sodium levels were $137.25 \pm 3.86 \mathrm{mmol} / \mathrm{L}$ and $137.56 \pm 2.97 \mathrm{mmol} / \mathrm{L}(P=0.799)$ for normotensive and preeclamptic pregnant women, respectively. Normotensive and preeclamptic pregnant women had a mean serum potassium of $3.66 \pm 0.66 \mathrm{mmol} / \mathrm{L}$ and 3.26 $\pm 0.23 \mathrm{mmol} / \mathrm{L}(P=0.029)$, respectively. Furthermore, mean serum chloride and mean serum bicarbonate levels were $108.25 \pm 4.83 \mathrm{mmol} / \mathrm{L}$ and $112.25 \pm 7.22$ $\mathrm{mmol} / \mathrm{L}(P=0.105)$, as well as $21.91 \pm 1.93 \mathrm{mmol} / \mathrm{L}$ and $22.34 \pm 2.43 \mathrm{mmol} / \mathrm{L}(P=0.577)$ for normotensive and preeclamptic pregnant women, respectively. Moreover, the mean serum urea levels were $5.29 \pm 2.10 \mathrm{mmol} / \mathrm{L}$ and $5.58 \pm 1.42 \mathrm{mmol} / \mathrm{L}(P=0.660)$ for normotensive and preeclamptic groups, respectively. The difference in the mean serum creatinine was significant $(P=0.010)$, representing $100.42 \pm 19.10 \mathrm{mmol} / \mathrm{L}$ and $131.50 \pm 40.75$ $\mathrm{mmol} / \mathrm{L}$ for normotensive women and pregnant women with preeclampsia, respectively.

Table 2 provides data on the correlation between systolic and diastolic BP with renal function parameters. The serum potassium had a significant negative relationship with systolic and diastolic BP $(r=0.480, P<0.01$ and $r=$ $0.391, P<0.05$, respectively). Finally, there was significant direct correlation between serum creatinine and systolic and diastolic BP $(r=0.535, P<0.01$ and $r=0.487, P<$ 0.01 , respectively).

\section{Discussion \\ Preeclampsia is a condition that is related to pregnancy and characterized by high BP and proteinuria, affecting multiple organs including the kidneys, liver, brain, and the blood clotting system (1-3). Based on the findings, there}


Table 1. Characteristics of the Study

\begin{tabular}{|c|c|c|c|c|}
\hline Characteristic & Groups & Mean Value & SD & $P$ Value \\
\hline \multirow{2}{*}{ Age } & Normotensive & 30.50 & 4.18 & \multirow[t]{2}{*}{0.904} \\
\hline & Preeclamptic & 30.69 & 4.54 & \\
\hline \multirow{2}{*}{ Systolic BP } & Normotensive & 114.38 & 9.64 & \multirow[t]{2}{*}{0.000} \\
\hline & Preeclamptic & 187.50 & 22.06 & \\
\hline \multirow{2}{*}{ Diastolic BP } & Normotensive & 76.25 & 5.00 & \multirow[t]{2}{*}{0.000} \\
\hline & Preeclamptic & 108.13 & 9.11 & \\
\hline \multirow{2}{*}{ BMI } & Normotensive & 28.14 & 2.85 & \multirow[t]{2}{*}{0.03} \\
\hline & Preeclamptic & 30.96 & 2.04 & \\
\hline \multirow{2}{*}{$\mathrm{Na}$} & Normotensive & 137.25 & 3.86 & \multirow[t]{2}{*}{0.799} \\
\hline & Preeclamptic & 137.56 & 2.97 & \\
\hline \multirow{2}{*}{ K } & Normotensive & 3.66 & 0.66 & \multirow[t]{2}{*}{0.029} \\
\hline & Preeclamptic & 3.26 & 0.23 & \\
\hline \multirow{2}{*}{$\mathrm{Cl}$} & Normotensive & 108.25 & 4.83 & \multirow[t]{2}{*}{0.105} \\
\hline & Preeclamptic & 112.25 & 7.22 & \\
\hline \multirow{2}{*}{$\mathrm{HCO}_{3}^{-}$} & Normotensive & 21.91 & 1.93 & \multirow[t]{2}{*}{0.577} \\
\hline & Preeclamptic & 22.34 & 2.43 & \\
\hline \multirow{2}{*}{ Urea } & Normotensive & 5.29 & 2.10 & \multirow[t]{2}{*}{0.660} \\
\hline & Preeclamptic & 5.58 & 1.42 & \\
\hline \multirow{2}{*}{ Creatinine } & Normotensive & 100.42 & 19.10 & \multirow[t]{2}{*}{0.010} \\
\hline & Preeclamptic & 131.50 & 40.75 & \\
\hline
\end{tabular}

Note. BP: Blood pressure; BMI: Body mass index; Na: Sodium; K: Potassium; Cl: Chloride; HCO3-: Bicarbonate.

was no significant change in the serum sodium between preeclamptic and normotensive pregnant women in the present study, which corroborates with the findings of Adewolu (9). Conversely, Manjareeka and Nanda observed a significantly higher mean serum sodium in preeclampsia (8). Although sodium retention has been proposed in preeclampsia, the pathophysiology is not clear. It may be due to the vasoconstriction of renal vessels leading to the reduction of the glomerular filtration rate and the stimulation of the renin-angiotensin-aldosterone mechanism (11). The serum sodium level did not significantly correlate with BP changes in this study, which is in line with the results of a previous study (9). This suggests that the serum sodium level could not be used as the marker of disease severity in preeclampsia in our environment.

The serum potassium was significantly lower in preeclampsia in this study, which also conforms with the finding of a previous study (8). The lower serum potassium in preeclampsia may be a reflection of an abnormal sodium/potassium transport system cell membrane of the vascular smooth muscle, which accounts for dysregulation in the BP (11). This study also observed a significant negative correlation between serum potassium and BP. On the other hand, Pikilidou et al reported an inverse relationship between the serum potassium level and BP among people with hypertension (12). Although the mechanism is unclear, potassium channels in vascular smooth muscle and endothelial cells regulate calcium entry into the cells, causing vasodilatation (13). Potassium also helps the renal excretion of sodium and water (14).

In the present study, no significant difference was observed in chloride and bicarbonate levels in preeclampsia when compared to normotensive pregnant women. Contrarily, Manjareeka and Nanda reported that the serum chloride and bicarbonate significantly elevated in preeclampsia (8). The changes in the serum chloride are linked to an alteration in the serum sodium levels as an increase in serum chloride levels results from a delay in the renal excretion of sodium (15). The serum chloride and bicarbonate in the present study demonstrated no significant relationship with $\mathrm{BP}$ changes.

Similarly, the serum urea showed no significant difference between preeclamptic and normotensive pregnant women although serum creatinine was significantly higher in preeclampsia, which is in conformity with the findings of a previous study (7). These changes may be due to an increase in the glomerular filtration resistance in preeclampsia by the mechanical effect of swelling in the cytoplasm, along with an alteration in the metabolism, leading to a reduction in renal perfusion and the glomerular filtration rate $(3,5)$. On the other hand, there was a significant positive relationship between serum creatinine and BP in this study. This may be explained by a reduction in creatinine excretion from the kidneys which is the cause or effect of an increase in renal vascular resistance, leading to elevated BP (5).

A well-designed study regarding the effect of preeclampsia on ion channels such as $\mathrm{Na}+\mathrm{K}+$ cotransporter may provide answers respecting the reasons for these changes and may proffer a solution for the prevention and a reduction in disease progression.

\section{Limitations of the Study}

This was a cross-sectional study. A longitudinal

Table 2. Pearson's Correlation Analysis

\begin{tabular}{lcccccc} 
& $\mathrm{Na}$ & $\mathrm{K}$ & $\mathrm{Cl}$ & $\mathrm{HCO}_{3}^{-}$ & Urea & Creatinine \\
\hline Systolic BB & 0.052 & $-0.480^{* *}$ & 0.268 & 0.170 & -0.104 \\
Diastolic BP & 0.080 & $-0.391^{*}$ & 0.325 & 0.198 & -0.107 & $0.485^{* *}$ \\
\hline
\end{tabular}

Note. Na: Sodium; K: Potassium; Cl: Chloride; $\mathrm{HCO}-:$ Bicarbonate.

${ }^{*}$ Correlation is significant at the $P<0.05$ level.

${ }^{* *}$ Correlation is significant at the $P<0.01$ level. 
study regarding renal parameters before and after the development of preeclampsia would provide more insights on biochemical changes.

\section{Conclusions}

The serum potassium and creatinine play significant roles in the pathogenesis and severity of preeclampsia. Other factors not highlighted in this study may also play essential roles in the disease progression. Therefore, the need for further studies on renal biochemical markers on preeclampsia is imperative.

\section{Conflict of Interests}

There was no conflict of interests among the authors during this research.

\section{Financial Support}

The authors received no external funding for this research.

\section{References}

1. Sibai B, Dekker G, Kupferminc M. Pre-eclampsia. Lancet. 2005;365(9461):785-799. doi:10.1016/s01406736(05)17987-2

2. Hutcheon JA, Lisonkova S, Joseph KS. Epidemiology of pre-eclampsia and the other hypertensive disorders of pregnancy. Best Pract Res Clin Obstet Gynaecol. 2011;25(4):391-403. doi:10.1016/j.bpobgyn.2011.01.006

3. Duley L. The global impact of pre-eclampsia and eclampsia. Semin Perinatol. 2009;33(3):130-137. doi:10.1053/j. semperi.2009.02.010

4. Kooffreh M, Ekott M, Ekpoudom D. The prevalence of pre-eclampsia among pregnant women in the University of Calabar Teaching Hospital, Calabar. Saudi Journal for Health Sciences. 2014;3(3):133-136. doi:10.4103/22780521.142317

5. Lafayette R. The kidney in preeclampsia. Kidney Int. 2005;67(3):1194-1203. doi:10.1111/j.1523-

\subsubsection{9.x}

6. Polsani S, Phipps E, Jim B. Emerging new biomarkers of preeclampsia. Adv Chronic Kidney Dis. 2013;20(3):271279. doi:10.1053/j.ackd.2013.01.001

7. Manjareeka M, Nanda S. Elevated levels of serum uric acid, creatinine or urea in preeclamptic women. Int J Med Sci Public Health. 2013;2(1):43-47. doi:10.5455/ ijmsph.2013.2.43-47

8. Manjareeka M, Nanda S. Serum electrolyte levels in preeclamptic women: a comparative study. Int J Pharma Bio Sci. 2012;3(2):572-578.

9. Adewolu O. Serum sodium, potassium, calcium and magnesium in women with pregnancy induced hypertension and preeclampsia in Oredo local Government, Benin Metropolis: a pilot study. Afr J Med Health Sci. 2013;12(1):1-5.

10. Grotegut CA. Prevention of preeclampsia. J Clin Invest. 2016;126(12):4396-4398. doi:10.1172/jci91300

11. Arumanayagam $M$, Rogers $M$. Platelet sodium pump and sodium potassium cotransport activity in nonpregnant, normotensive, and hypertensive pregnant women. Hypertens Pregnancy. 1999;18(1):35-44. doi:10.3109/10641959909009609

12. Pikilidou MI, Lasaridis AN, Sarafidis PA, et al. Blood pressure and serum potassium levels in hypertensive patients receiving or not receiving antihypertensive treatment. Clin Exp Hypertens. 2007;29(8):563-573. doi:10.1080/10641960701744103

13. Salomonsson M, Brasen JC, Sorensen CM. Role of renal vascular potassium channels in physiology and pathophysiology. Acta Physiol (Oxf). 2017;221(1):14-31. doi:10.1111/apha.12882

14. Palygin O, Levchenko V, Ilatovskaya DV, et al. Essential role of Kir5.1 channels in renal salt handling and blood pressure control. JCI Insight. 2017;2(18). doi:10.1172/jci. insight.92331

15. Shrimanker I, Bhattarai S. Electrolytes. StatPearls. Treasure Island, FL: StatPearls Publishing; 2019

(c) 2020 The Author(s); This is an open-access article distributed under the terms of the Creative Commons Attribution License (http:// creativecommons.org/licenses/by/4.0), which permits unrestricted use, distribution, and reproduction in any medium, provided the original work is properly cited. 